\title{
Overweight and obesity prevalence among Cree youth of Eeyou Istchee according to three body mass index classification systems
}

\author{
Audray St-Jean ${ }^{1}$, Salma Meziou', Pierre Ayotte ${ }^{1,2,3}$ and Michel Lucas ${ }^{1,3^{*}}$ (D)
}

\begin{abstract}
Background: Little is known about the suitability of three commonly used body mass index (BMI) classification systems for Indigenous youth. We estimated overweight and obesity prevalence among Cree youth of Eeyou Istchee according to three BMI classification systems, assessed the level of agreement between them, and evaluated their accuracy through body fat and cardiometabolic risk factors.

Methods: Data on 288 youth (aged 8-17 years) were collected. Overweight and obesity prevalence were estimated with Centers for Disease Control and Prevention (CDC), International Obesity Task Force (IOTF) and World Health Organization (WHO) criteria. Agreement was measured with weighted kappa (kw). Associations with body fat and cardiometabolic risk factors were evaluated by analysis of variance.

Results: Obesity prevalence was $42.7 \%$ with IOTF, 47.2\% with CDC, and 49.3\% with WHO criteria. Agreement was almost perfect between IOTF and CDC ( $\mathrm{KW}=0.93)$, IOTF and WHO ( $\mathrm{kW}=0.91)$, and WHO and CDC $(\mathrm{KW}=0.94)$. Means of body fat and cardiometabolic risk factors were significantly higher $\left(P_{\text {trend }}<0.001\right)$ from normal weight to obesity, regardless of the system used. Youth considered overweight by IOTF but obese by CDC or WHO exhibited less severe clinical obesity.
\end{abstract}

Conclusions: IOTF seems to be more accurate in identifying obesity in Cree youth.

Keywords: Body mass index, Youth, Obesity, Overweight, Eeeyou Istchee Cree

\section{Background}

Overweight and obesity are defined as abnormal or excessive fat accumulation that may impair health [1]. Associations are well-documented for overweight and obesity in childhood and adolescence with cardiovascular and metabolic complications as well as premature mortality in adulthood $[2,3]$. The high prevalence of obesity among youth has become a major public health

\footnotetext{
* Correspondence: michel.lucas@crchuq.ulaval.ca

'Population Health and Optimal Health Practices Research Unit, CHU de Québec - Université Laval, Hôpital du Saint-Sacrement, 1050 Chemin Sainte-Foy, Québec, QC G1S 4L8, Canada

${ }^{3}$ Department of Social and Preventive Medicine, Faculty of Medecine,

Université Laval, 1050 Avenue de la Médecine, Local 2428, Québec, QC G1V

06A, Canada

Full list of author information is available at the end of the article
}

issue [4]. In Canada, obesity is more pervasive among Indigenous than non-Indigenous youth $[5,6]$.

Body mass index (BMI) is the most common method of assessing weight status and health risks in youth. Because BMI varies with growth and maturation during childhood and adolescence, age- and sex-specific cut-off points are needed for appropriate overweight and obesity classification. Three BMI classification systems are commonly used to study youth, with cut-off values published by the International Obesity Task Force (IOTF) [7], the Centers for Disease Control and Prevention (CDC) [8], and the World Health Organization (WHO) [9]. IOTF cut-offs are derived from six large surveys conducted in Brazil, UK, Hong Kong, the Netherlands, Singapore, and USA. CDC growth references are based on data from five nationally representative surveys of American youth. 
WHO growth curves are drawn from the WHO Multicentre Growth Reference Study conducted in six countries (Brazil, Ghana, India, Norway, Oman, and USA).

Inconsistent overweight and obesity prevalence estimation according to these classification systems poses challenges. In the same population, estimates tend to be the lowest for IOTF whereas WHO cut-offs appear to be the highest [10-13]. Our team previously studied prevalence estimates of overweight and obesity among Inuit youth according to IOTF, CDC and WHO criteria [14]. These BMI classification systems were based on populations that did not include Indigenous youth. The Cree are a unique cultural and ethnic group. Therefore, it is particularly interesting to verify the suitability of such BMI systems in this population.

The present study estimated overweight and obesity prevalence among Cree youth of Eeyou Istchee, northern Quebec according to three BMI classification systems, assessed the level of agreement between these classification systems, and evaluated their accuracy with body fat percentage and cardiometabolic risk factors as surrogates of obesity-related outcomes.

\section{Methods}

\section{Study design and population}

Data were sourced from the cross-sectional "Nituuchischaayihtitaau Aschii: A Multi-Community Environment-and-Health Study in Eeyou Istchee", a collaboration of the Cree Board of Health and Social Services of James Bay (CBHSSJB) with Laval, McGill and McMaster Universities. The study design has been described previously [15]. Briefly, a random sample of participants was recruited from seven communities of Eeyou Istchee (latitude $>49.6^{\circ} \mathrm{N}$ ). Data were collected during the spring and/or summer of 2005, 2007, 2008 and 2009. Participants were advised to fast overnight and, during the next day's appointment, a research nurse measured anthropometric data and collected venous blood samples which were kept frozen at $-80{ }^{\circ} \mathrm{C}$ and transported to the CHUQ Research Centre, Québec (Canada), for biological analysis. Inclusion criteria for the present analysis were age 8-17 years and blood samples collected under fasting conditions $(\geq 8 \mathrm{~h})$. The study population also included participants aged between 0 and 7 years old. However, anthropometric and clinical measurements were not assessed in this age group. Two of the initial 290 participants selected were excluded because of missing BMI data, leaving 288 participants for analysis.

Participation was voluntary, and written informed consent was given by one of the child's parents or guardian. Ethics approval was obtained from all participating institutions.

\section{Anthropometric data}

Weight without shoes was measured with a bioelectrical impedance scale (Tanita Corp., Arlington Heights, IL, USA). Height without shoes was quantified using a measuring tape with patients standing barefoot on a hard surface. Waist circumference (WC) was assessed at the end of exhalation by tape located midway between the lower margin of the last floating rib and the top of the iliac crest [1]. Height was recorded to the nearest $\mathrm{cm}$, and $\mathrm{WC}$, to the nearest $0.5 \mathrm{~cm}$. BMI was calculated by dividing weight $(\mathrm{kg})$ by squared height $\left(\mathrm{m}^{2}\right)$.

Weight status was defined according to 2005 IOTF [7], $2000 \mathrm{CDC}$ [8], and $2007 \mathrm{WHO}$ criteria [9]. IOTF cut-off values are age- and sex-specific extrapolations of adult overweight $\left(\mathrm{BMI} \geq 25 \mathrm{~kg} / \mathrm{m}^{2}\right)$ and obesity $\left(B M I \geq 30 \mathrm{~kg} / \mathrm{m}^{2}\right)$ definitions at 18 years. CDC BMI age- and sex-specific growth references classify overweight as $85^{\text {th }}$ percentile $\leq \mathrm{BMI}<95^{\text {th }}$ percentile, and obesity, as $\mathrm{BMI} \geq 95^{\text {th }}$ percentile. WHO BMI-for-age categorizes overweight as $\mathrm{BMI}>+1$ standard deviation above

Table 1 Characteristics of study participants (8-17 years) from Eeyou Istchee communities of northern Quebec, Canada, 2005-2009

\begin{tabular}{lllll}
\hline & $\begin{array}{l}\text { Total } \\
(n=288)\end{array}$ & $\begin{array}{l}\text { Boys } \\
(n=143)\end{array}$ & $\begin{array}{l}\text { Girls } \\
(n=145)\end{array}$ & $P$ \\
\hline Age, years & $12.4 \pm 2.7$ & $12.7 \pm 2.7$ & $12.1 \pm 2.8$ & 0.10 \\
Anthropometric data & & & & \\
Height, cm & $159 \pm 13.6$ & $162 \pm 14.7$ & $156 \pm 11.7$ & $<0.001$ \\
Weight, kg & $64.7 \pm 22.6$ & $67.5 \pm 23.7$ & $62.0 \pm 21.2$ & 0.04 \\
WC, cm & $88.3 \pm 15.7$ & $89.9 \pm 16.1$ & $86.7 \pm 15.2$ & 0.08 \\
BMl, kg/m ${ }^{2}$ & $25.1 \pm 6.1$ & $25.1 \pm 5.9$ & $25.0 \pm 6.2$ & 0.93 \\
BMl z-score & $1.3 \pm 1.0$ & $1.3 \pm 1.0$ & $1.3 \pm 1.0$ & 0.46 \\
Body fat, \% & $32.5 \pm 10.9$ & $29.0 \pm 11.4$ & $36.1 \pm 9.1$ & $<0.001$ \\
Cardiometabolic risk factors & & & \\
SBP, mm Hg & $105 \pm 12.1$ & $107 \pm 12.8$ & $103 \pm 11.0$ & 0.01 \\
DBP, mm Hg & $62 \pm 10.6$ & $63 \pm 11.1$ & $61 \pm 10.1$ & 0.09 \\
Fasting plasma & $5.1 \pm 0.44$ & $5.1 \pm 0.42$ & $5.0 \pm 0.46$ & 0.05 \\
glucose, mmol/L & & & & \\
Fasting plasma & $145 \pm 109$ & $129 \pm 95.7$ & $160 \pm 120$ & 0.02 \\
insulin, pmol/L & & & & \\
HOMA2-IR & $2.5 \pm 1.7$ & $2.3 \pm 1.4$ & $2.8 \pm 1.8$ & 0.008 \\
TG, mmol/L & $1.0 \pm 0.51$ & $0.90 \pm 0.44$ & $1.0 \pm 0.58$ & 0.07 \\
HDL-C, mmol/L & $1.3 \pm 0.32$ & $1.3 \pm 0.33$ & $1.3 \pm 0.31$ & 0.70 \\
TG/HDL-C ratio & $0.84 \pm 0.60$ & $0.78 \pm 0.50$ & $0.89 \pm 0.69$ & 0.22 \\
\hline
\end{tabular}

Values are presented as arithmetic mean \pm standard deviation. $P$ values were obtained with two-sided $t$-tests

Information on fasting plasma glucose and insulin was missing for 1 participant, on HOMA2-IR for 3 participants, and on TG, HDL-C and TG/HDL-C ratio for 91 participants

$B M I$ body mass index; $D B P$ diastolic blood pressure; $H D L-C$ high-density lipoprotein cholesterol; HOMA2-IR homeostatic model assessment 2 of insulin resistance; $S B P$ systolic blood pressure; $T G$ triglycerides; WC waist circumference 
the WHO growth standard median while BMI $>+2$ standard deviations is considered as obesity. Participants who were neither overweight nor obese were defined as normal weight. BMI z-scores were calculated by the CDC SAS program [16]. Body fat percentage (\%) was assessed by bioelectrical impedance analyzer (Tanita TBF-300, GHT Canada, Laval, QC, Canada).

\section{Cardiometabolic risk factors}

Systolic (SBP) and diastolic blood pressure (DBP) were measured according to Canadian Hypertensive Education Program recommendations [17]. Fasting plasma glucose was quantified by spectrophotometric assay (Vitros 950 Chemistry Station, Ortho-Clinical Diagnostics, Raritan, NJ, USA), and fasting plasma insulin, by immunoassay with chemiluminescent detection (Advia Centaur, Siemens, Washington, DC, USA). Homeostatic model assessment 2 of insulin resistance (HOMA2-IR) was calculated from fasting plasma glucose and insulin levels [18]. Triglycerides (TG) and high-density lipoprotein cholesterol (HDL-C) were evaluated by enzymatic methods (Vitros 950 Chemistry Station, Ortho-Clinical Diagnostics). TG/HDL-C ratio was ascertained by dividing TG by HDL-C concentrations. Lipids were not assessed in participants aged 8 to 14 years during the 2005 (1 community) and 2007 (2 communities) surveys, which corresponded to missing values for 91 participants.

\section{Statistical analyses}

The characteristics of study participants per gender were compared by $t$-tests and reported as arithmetic means \pm standard deviations. Overweight and obesity prevalence estimates (\%) according to the three BMI classification systems were presented graphically, and differences between prevalence rates were compared by chi-square tests. Agreements between BMI classification systems were evaluated by weighted kappa ( $\mathrm{kW}$ ) coefficients according to Landis and Koch's guiding principles (19). $\mathrm{KW}$ coefficients between 0 and 0.20 are considered as slight, $0.21-0.40$ as fair, $0.41-0.60$ as moderate, $0.61-0.80$ as substantial, and $0.81-1.00$ as almost perfect [19].

A

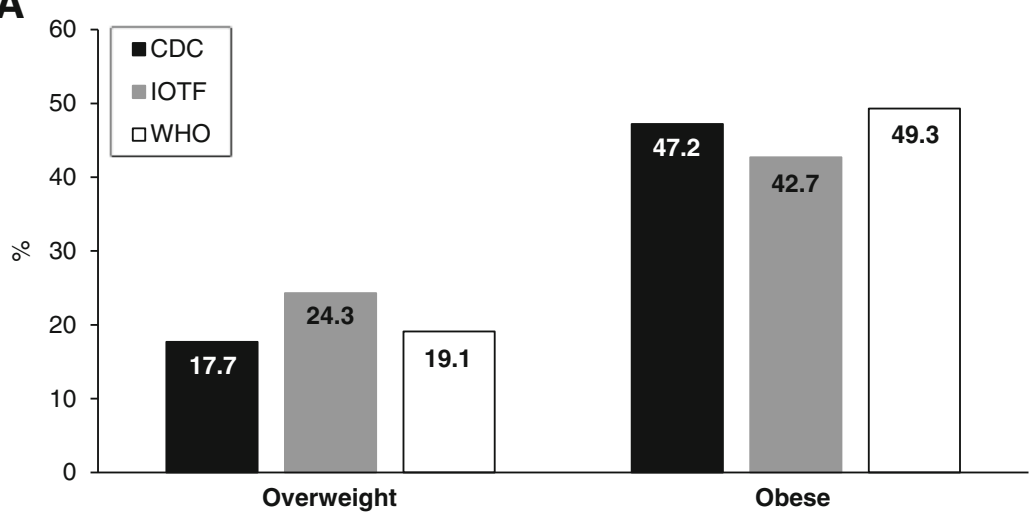

B

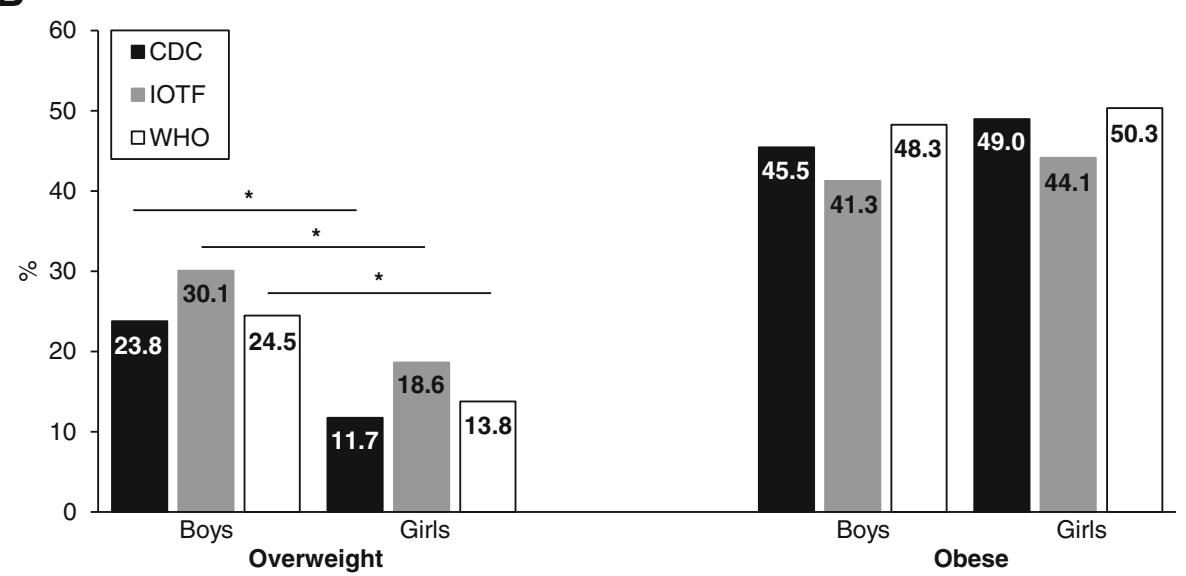

Fig. 1 Overweight and obesity prevalence (a): whole study sample, (b): by gender) according to IOTF, CDC, and WHO classification systems among youth from Eeyou Istchee communities of northern Quebec, Canada, 2005-2009. Values are presented as prevalence (\%). * Statistically different from girls $(P<0.05)$. CDC, Centers for Disease Control and Prevention; IOTF, International Obesity Task Force; WHO, World Health Organization 
Analysis of variance was used to investigate whether means of body fat and cardiometabolic risk factors were different according to weight status. Tests for trend were assessed by assigning the median BMI value to each weight status category and modelling this value as a continuous variable using the contrast statement of the SAS PROC GLM procedure. Mean differences in body fat and cardiometabolic risk factors according to agreement and or non-agreement between weight status based on IOTF/CDC and IOTF/WHO classification systems were calculated. All statistical analyses were performed with SAS software (version 9.4, SAS Institute Inc., Cary, NC, USA). Two-sided $P<0.05$ values were considered to be statistically significant.

\section{Results}

Characteristics of study participants from the seven Cree communities are presented by gender in Table 1 . Mean age was 12.4 years (range $8-17$ years), $50.3 \%$ were girls, and mean BMI z-score was 1.3. Differences between the two genders were statistically significant for numerous variables. Girls had significantly higher body fat, fasting plasma insulin and HOMA2-IR score than boys. Weight, height and SBP were significantly lower among girls compared to boys.

Prevalence estimates of overweight and obesity according to each BMI classification system are presented for the whole study sample (Fig. 1a) and by gender (Fig. 1b). Overall overweight prevalence was $24.3 \%$ with IOTF, $17.7 \%$ with CDC, and $19.1 \%$ with WHO criteria. The percentages of youth categorized as obese were $42.7 \%, 47.2 \%$, and $49.3 \%$ respectively per IOTF, $\mathrm{CDC}$, and WHO criteria. Overweight prevalence estimates were significantly higher $(P<0.05)$ among boys (11.5\% with IOTF, $12.1 \%$ with $\mathrm{CDC}$, and $10.7 \%$ with WHO) than girls. Overweight prevalence rates appeared to be greater with IOTF criteria regardless of gender, whereas $\mathrm{CDC}$ and WHO proportions were similar in boys and girls. Higher estimates of obesity were obtained for both genders with WHO and CDC systems compared to IOTF. However, these differences were not statistically significant. Level of agreement between weight status categories based on the three BMI classification systems are reported in Table 2. $\mathrm{kW}$ coefficients indicated almost perfect agreement between IOTF and CDC ( $\mathrm{Kw}=0.93,95 \% \mathrm{CI}: 0.90-0.96$; 93.41\% agreement), IOTF and WHO ( $\mathrm{kw}=0.91,95 \%$ CI: $0.87-0.94 ; 91.32 \%$ agreement), and WHO and CDC ( $\mathrm{KW}=0.94,95 \%$ CI: $0.91-0.97 ; 94.45 \%$ agreement).

Means of body fat and cardiometabolic risk factors by weight status and BMI classification systems are enumerated in Table 3. Body fat percentages were significantly higher from normal weight to obesity for IOTF, CDC, and WHO criteria (all $P_{\text {trend }}<0.001$ ). All
Table 2 Agreements between weight status based on IOTF, $\mathrm{CDC}$ and $\mathrm{WHO}$ classification systems

\begin{tabular}{lllll}
\hline & \multicolumn{1}{l}{ CDC } & & \\
\cline { 2 - 4 } OTF & Normal weight & Overweight & Obese & Total \\
\hline Normal weight & $95(32.99 \%)$ & 0 & 0 & 95 \\
Overweight & $6(2.08 \%)$ & $51(17.71 \%)$ & $13(4.51 \%)$ & 70 \\
Obese & 0 & 0 & $123(42.71 \%)$ & 123 \\
Total & 101 & 51 & 136 & 288 \\
& WHO & & & \\
IOTF & Normal weight & Overweight & Obese & Total \\
Normal weight & $90(31.25 \%)$ & $5(1.74 \%)$ & 0 & 95 \\
Overweight & $1(0.35 \%)$ & $50(17.36 \%)$ & $19(6.60 \%)$ & 70 \\
Obese & 0 & 0 & $123(42.71 \%)$ & 123 \\
Total & 91 & 55 & 142 & 288 \\
& CDC & & & \\
WHO & Normal weight & Overweight & Obese & Total \\
Normal weight & $91(31.60 \%)$ & 0 & 0 & 91 \\
Overweight & $10(3.47 \%)$ & $45(15.63 \%)$ & 0 & 55 \\
Obese & 0 & $6(2.08 \%)$ & $136(47.22 \%)$ & 142 \\
Total & 101 & 51 & 136 & 288 \\
\hline
\end{tabular}

$\%$ of agreement $=91.32 \% ; \mathrm{KW}=0.91,95 \% \mathrm{Cl}: 0.87-0.94 ; P<0.001$

$\%$ of agreement $=94.45 \% ; \mathrm{KW}=0.94,95 \% \mathrm{Cl}: 0.91-0.97 ; P<0.001$

$\%$ of agreement was calculated by adding concordant percentages CDC Centers for Disease Control and Prevention; $\mathrm{Cl}$ confidence interval; IOTF International Obesity Task Force; $K W$ weighted Kappa;

WHO World Health Organization

cardiometabolic risk factors except HDL-C presented the same pattern. SBP, DBP, fasting plasma glucose, fasting plasma insulin, HOMA2-IR, TG and TG/HDL-C ratio were significantly higher with weight status shift escalation, regardless of BMI system (all $P_{\text {trend }}<0.05$ ), whereas HDL-C concentrations were significantly lower from normal weight to obesity $\left(P_{\text {trend }}<0.001\right)$.

Table 2 depicts mean differences of body fat and cardiometabolic risk factors in non-agreement youth (i.e., those considered overweight by IOTF, but obese by $\mathrm{CDC}$ or WHO criteria) compared to agreement youth (i.e., those classified as obese by both IOTF and CDC or IOTF and WHO cut-offs). Non-agreement youth exhibited significantly lower means $(P<0.05)$ of body fat, fasting plasma insulin and HOMA2-IR score than agreement youth. Non-agreement youth with WHO cutoffs also had significantly higher $(P<0.05)$ HDL-C levels than agreement youth.

\section{Discussion}

We observed high prevalence rates of overweight and obesity regardless of the growth reference used. WHO cut-offs generated the highest prevalence estimates of overweight and obesity for participants overall and for both genders compared to CDC and IOTF. Despite a good level of agreement observed 
Table 3 Means of body fat percentage and cardiometabolic risk factors according to IOTF, CDC and WHO classification systems

\begin{tabular}{|c|c|c|c|c|}
\hline & Normal weight & Overweight & Obese & $P_{\text {trend }}$ \\
\hline & IOTF & & & \\
\hline & $(n=95)$ & $(n=70)$ & $(n=123)$ & \\
\hline Body fat, $\%$ & $22.8 \pm 7.5^{1}$ & $29.7 \pm 7.1^{2}$ & $41.7 \pm 6.7^{3}$ & $<0.001$ \\
\hline $\mathrm{SBP}, \mathrm{mm} \mathrm{Hg}$ & $102 \pm 12.8^{1}$ & $103 \pm 10.6^{1}$ & $108 \pm 11.6^{2}$ & $<0.001$ \\
\hline $\mathrm{DBP}, \mathrm{mm} \mathrm{Hg}$ & $60 \pm 9.8^{1}$ & $61 \pm 8.7^{1}$ & $65 \pm 11.6^{2}$ & $<0.001$ \\
\hline Fasting plasma glucose, mmol/L & $5.0 \pm 0.40^{1}$ & $5.1 \pm 0.35^{1,2}$ & $5.2 \pm 0.50^{2}$ & 0.005 \\
\hline Fasting plasma insulin, pmol/L & $86.4 \pm 28.5^{1}$ & $116 \pm 62.8^{1}$ & $207 \pm 135^{2}$ & $<0.001$ \\
\hline HOMA2-IR & $1.6 \pm 0.52^{1}$ & $2.1 \pm 1.1^{1}$ & $3.5 \pm 2.0^{2}$ & $<0.001$ \\
\hline $\mathrm{TG}, \mathrm{mmol} / \mathrm{L}$ & $0.76 \pm 0.28^{1}$ & $0.90 \pm 0.46^{1}$ & $1.1 \pm 0.61^{2}$ & $<0.001$ \\
\hline $\mathrm{HDL}-\mathrm{C}, \mathrm{mmol} / \mathrm{L}$ & $1.5 \pm 0.26^{1}$ & $1.3 \pm 0.32^{2}$ & $1.1 \pm 0.28^{3}$ & $<0.001$ \\
\hline TG/HDL-C ratio & $0.55 \pm 0.28^{1}$ & $0.7 \pm 0.43^{1}$ & $1.1 \pm 0.72^{2}$ & $<0.001$ \\
\hline & CDC & & & \\
\hline & $(n=101)$ & $(n=51)$ & $(n=136)$ & \\
\hline Body fat, $\%$ & $23.2 \pm 7.4^{1}$ & $28.5 \pm 7.1^{2}$ & $41.0 \pm 7.0^{3}$ & $<0.001$ \\
\hline $\mathrm{SBP}, \mathrm{mm} \mathrm{Hg}$ & $102 \pm 12.6^{1}$ & $102 \pm 10.9^{1}$ & $108 \pm 11.5^{2}$ & $<0.001$ \\
\hline $\mathrm{DBP}, \mathrm{mm} \mathrm{Hg}$ & $60 \pm 9.8^{1}$ & $60 \pm 8.4^{1}$ & $65 \pm 11.3^{2}$ & $<0.001$ \\
\hline Fasting plasma glucose, mmol/L & $5.0 \pm 0.39^{1}$ & $5.1 \pm 0.35^{1,2}$ & $5.2 \pm 0.50^{2}$ & 0.002 \\
\hline Fasting plasma insulin, pmol/L & $86.3 \pm 28.5^{1}$ & $114 \pm 67.0^{1}$ & $201 \pm 131^{2}$ & $<0.001$ \\
\hline HOMA2-IR & $1.6 \pm 0.52^{1}$ & $2.1 \pm 1.2^{1}$ & $3.5 \pm 1.9^{2}$ & $<0.001$ \\
\hline $\mathrm{TG}, \mathrm{mmol} / \mathrm{L}$ & $0.77 \pm 0.28^{1}$ & $0.87 \pm 0.48^{1}$ & $1.1 \pm 0.60^{2}$ & $<0.001$ \\
\hline $\mathrm{HDL}-\mathrm{C}, \mathrm{mmol} / \mathrm{L}$ & $1.5 \pm 0.26^{1}$ & $1.3 \pm 0.34^{1}$ & $1.1 \pm 0.29^{2}$ & $<0.001$ \\
\hline TG/HDL-C ratio & $0.56 \pm 0.27^{1}$ & $0.70 \pm 0.44^{1}$ & $1.1 \pm 0.71^{2}$ & $<0.001$ \\
\hline & WHO & & & \\
\hline & $(n=91)$ & $(n=55)$ & $(n=142)$ & \\
\hline Body fat, $\%$ & $22.8 \pm 7.5^{1}$ & $28.0 \pm 6.9^{2}$ & $40.6 \pm 7.2^{3}$ & $<0.001$ \\
\hline $\mathrm{SBP}, \mathrm{mm} \mathrm{Hg}$ & $103 \pm 13.0^{1}$ & $102 \pm 10.9^{1}$ & $107 \pm 11.4^{2}$ & 0.001 \\
\hline $\mathrm{DBP}, \mathrm{mm} \mathrm{Hg}$ & $59 \pm 9.9^{1}$ & $60 \pm 7.9^{1}$ & $65 \pm 11.4^{2}$ & $<0.001$ \\
\hline Fasting plasma glucose, mmol/L & $5.0 \pm 0.40^{1}$ & $5.0 \pm 0.35^{1,2}$ & $5.2 \pm 0.49^{2}$ & 0.008 \\
\hline Fasting plasma insulin, pmol/L & $85.8 \pm 28.0^{1}$ & $109 \pm 62.9^{1}$ & $197 \pm 130^{2}$ & $<0.001$ \\
\hline HOMA2-IR & $1.6 \pm 0.51^{1}$ & $2.0 \pm 1.1^{1}$ & $3.4 \pm 1.9^{2}$ & $<0.001$ \\
\hline $\mathrm{TG}, \mathrm{mmol} / \mathrm{L}$ & $0.77 \pm 0.29^{1}$ & $0.86 \pm 0.46^{1}$ & $1.1 \pm 0.59^{2}$ & $<0.001$ \\
\hline $\mathrm{HDL}-\mathrm{C}, \mathrm{mmol} / \mathrm{L}$ & $1.5 \pm 0.27^{1}$ & $1.3 \pm 0.32^{1}$ & $1.2 \pm 0.30^{2}$ & $<0.001$ \\
\hline TG/HDL-C ratio & $0.55 \pm 0.28^{1}$ & $0.70 \pm 0.43^{1}$ & $1.1 \pm 0.70^{2}$ & $<0.001$ \\
\hline
\end{tabular}

Values are presented as arithmetic means \pm standard deviations. Means across weight status categories were compared by Scheffe's tests, and $P_{\text {trend }}$ was assessed by linear contrast

Information on fasting plasma glucose and insulin was missing for 1 participant, on HOMA2-IR for 3 participants and on TG, HDL-C and TG/HDL-C ratio for 91 participants

CDC Centers for Disease Control and Prevention; DBP diastolic blood pressure; HDL-C high-density lipoprotein cholesterol; HOMA2-IR homeostatic model assessment 2 of insulin resistance; IOTF International Obesity Task Force; SBP systolic blood pressure; TG triglycerides; WC waist circumference; WHO World Health Organization

${ }_{1,2,3}$,Values with different superscript numbers are statistically different $(P<0.05)$

between these BMI classification systems using the weighted kappa statistic, we noted significant differences for discordant obesity. Youth, who were considered overweight by IOTF classification but not by CDC or WHO (non-agreement), exhibited less severe clinical obesity - characterized by lower levels of body fat, insulin and HOMA2-IR score.
Several studies, using these three classification systems, have reported inconsistent prevalence estimates of overweight and obesity among youth worldwide [13, 20-26]. Overall, WHO criteria yielded the highest overweight and obesity prevalence. We noted overweight/obesity prevalence of $67.0 \%$ with IOTF, $64.9 \%$ with CDC, and $68.4 \%$ with WHO criteria. Among Canadian youth, prevalence 
for the combined overweight/obesity category (5-17 years) was estimated to be $24.8 \%$ ( $8.4 \%$ obesity) with IOTF and $31.5 \%$ (11.7\% obesity) with WHO [27]. Our results are similar to those of our previous study of Inuit youth from Nunavik [14]. Lower prevalence estimates of obesity were observed with IOTF and CDC compared to WHO criteria, which generated higher values regardless of gender. In other studies where IOTF generated the lowest estimates of both overweight and obesity prevalence [10-13], a higher prevalence of overweight was apparent for participants overall and by gender with IOTF compared to the $\mathrm{CDC}$ and WHO systems.

To investigate the accuracy of these three BMI classification systems in our population and in the absence of obesity-related outcomes (Table 4), we used body fat and different cardiometabolic risk factors [28] as surrogates. All variables were significantly higher whereas HDL-C levels were significantly lower from normal weight to obesity regardless of the BMI classification system. Our previous results on Inuit youth disclosed similar patterns for plasma insulin and high-sensitivity $\mathrm{C}$-reactive protein concentrations for the three growth references. Adiponectin levels were also significantly higher but only with WHO classification.

In our sample, IOTF criteria appear to be more accurate than $\mathrm{CDC}$ or WHO in identifying youth with obesity. According to the IOTF classification system, $90.4 \%$ of cases with obesity would be classified as obese with CDC and $86.6 \%$ with WHO. Also, we observed that youth classified as overweight by IOTF but not by CDC or WHO exhibited less severe clinical obesity. The lower proportion of subjects with obesity identified by IOTF was reflected by higher classification in the overweight category compared to $\mathrm{CDC}$ or WHO. In other words, false-positive subjects with obesity identified by CDC or WHO criteria were adequately classified as overweight by IOTF.

Our study has some limitations. Because of limitations inherent in cross-sectional investigations, no causal relationship can be ascertained. The cross-sectional design did not provide information on weight status variations

Table 4 Mean difference of body fat percentage and cardiometabolic risk factors according to agreement and non-agreement between weight status based on IOTF and CDC, and IOTF and WHO classification systems

\begin{tabular}{|c|c|c|c|c|}
\hline & \multicolumn{4}{|l|}{ IOTF vs. CDC } \\
\hline & $\begin{array}{l}\text { Non-agreement IOTF overweight/ } \\
\text { CDC obese }(n=13)\end{array}$ & $\begin{array}{l}\text { Agreement IOTF obese/ } \\
\text { CDC obese }(n=123)\end{array}$ & $\begin{array}{l}\text { Mean difference } \\
(95 \% \mathrm{Cl})\end{array}$ & $P$ \\
\hline Body fat, $\%$ & $34.5 \pm 6.6$ & $41.7 \pm 6.7$ & $-7.1(-11.0,-3.2)$ & $<0.001$ \\
\hline $\mathrm{SBP}, \mathrm{mm} \mathrm{Hg}$ & $104 \pm 10.4$ & $108 \pm 11.6$ & $-3.5(-10.2,3.1)$ & 0.29 \\
\hline $\mathrm{DBP}, \mathrm{mm} \mathrm{Hg}$ & $63 \pm 8.9$ & $65 \pm 11.6$ & $-2.1(-8.7,4.4)$ & 0.52 \\
\hline Fasting plasma glucose, mmol/L & $5.0 \pm 0.40$ & $5.2 \pm 0.50$ & $-0.17(-0.45,0.12)$ & 0.26 \\
\hline Fasting plasma insulin, pmol/L & $142 \pm 49$ & $207 \pm 135$ & $-65.3(-102,-28.5)$ & 0.001 \\
\hline HOMA2-IR & $2.6 \pm 0.86$ & $3.5 \pm 2.0$ & $-0.97(-1.6,-0.36)$ & 0.003 \\
\hline $\mathrm{TG}, \mathrm{mmol} / \mathrm{L}$ & $1.1 \pm 0.45$ & $1.1 \pm 0.61$ & $0.003(-0.50,0.51)$ & 0.99 \\
\hline $\mathrm{HDL}-\mathrm{C}, \mathrm{mmol} / \mathrm{L}$ & $1.2 \pm 0.32$ & $1.1 \pm 0.28$ & $0.11(-0.13,0.35)$ & 0.38 \\
\hline \multirow[t]{3}{*}{ TG/HDL-C ratio } & $0.97 \pm 0.46$ & $1.1 \pm 0.72$ & $-0.14(-0.74,0.46)$ & 0.64 \\
\hline & \multicolumn{4}{|l|}{ IOTF vs. WHO } \\
\hline & $\begin{array}{l}\text { Non-agreement IOTF overweight/ } \\
\text { WHO obese }(n=19)\end{array}$ & $\begin{array}{l}\text { Agreement IOTF obese/ } \\
\text { WHO obese }(n=123)\end{array}$ & $\begin{array}{l}\text { Mean difference } \\
(95 \% \mathrm{Cl})\end{array}$ & $P$ \\
\hline Body fat, $\%$ & $33.6 \pm 6.5$ & $41.7 \pm 6.7$ & $-8.1(-11.3,-4.8)$ & $<0.001$ \\
\hline $\mathrm{SBP}, \mathrm{mm} \mathrm{Hg}$ & $104 \pm 9.3$ & $108 \pm 11.6$ & $-4.2(-9.7,1.3)$ & 0.13 \\
\hline $\mathrm{DBP}, \mathrm{mm} \mathrm{Hg}$ & $63 \pm 10.1$ & $65 \pm 11.6$ & $-2.2(-7.7,3.4)$ & 0.44 \\
\hline Fasting plasma glucose, $\mathrm{mmol} / \mathrm{L}$ & $5.1 \pm 0.39$ & $5.2 \pm 0.50$ & $-0.12(-0.36,0.12)$ & 0.32 \\
\hline Fasting plasma insulin, pmol/L & $135 \pm 55.3$ & $207 \pm 135$ & $-71.9(-107,-36.6)$ & $<0.001$ \\
\hline HOMA2-IR & $2.5 \pm 0.98$ & $3.5 \pm 2.0$ & $-1.1(-1.7,-0.50)$ & $<0.001$ \\
\hline $\mathrm{TG}, \mathrm{mmol} / \mathrm{L}$ & $0.97 \pm 0.40$ & $1.1 \pm 0.61$ & $-0.18(-0.55,0.20)$ & 0.36 \\
\hline $\mathrm{HDL}-\mathrm{C}, \mathrm{mmol} / \mathrm{L}$ & $1.4 \pm 0.35$ & $1.1 \pm 0.28$ & $0.23(0.04,0.42)$ & 0.02 \\
\hline TG/HDL-C ratio & $0.77 \pm 0.43$ & $1.1 \pm 0.72$ & $-0.34(-0.78,0.10)$ & 0.13 \\
\hline
\end{tabular}

Values are presented as arithmetic means \pm standard deviations. $P$ values were obtained by $t$-tests

Information on fasting plasma glucose and insulin was missing for 1 participant, on HOMA2-IR for 3 participants, and on TG, HDL-C and TG/HDL-C ratio for 91 participants

$C D C$ Centers for Disease Control and Prevention; DBP diastolic blood pressure; HDL-C high-density lipoprotein cholesterol; HOMA2-IR homeostatic model assessment 2 for insulin resistance; IOTF International Obesity Task Force; SBP systolic blood pressure; TG triglycerides; WHO World Health Organization 
through age and timing growth patterns. As the study participants were Indigenous, with a high prevalence of overweight and obesity, generalizability of the observed associations may be limited to similar populations. The strengths of this study are its relatively large sample and direct assessment of weight, height, body fat and large number of biological measurements under fasting conditions, especially cardiometabolic risk factors. These allowed us to investigate the accuracy of the three BMI classification systems to estimate overweight and obesity prevalence in our population.

\section{Conclusions}

In summary, IOTF criteria seem to be more suitable than $\mathrm{CDC}$ and WHO in identifying more severe clinical obesity in our sample of Cree youth of Eeyou Istchee, northern Quebec. IOTF criteria generated lower obesity prevalence estimates than $\mathrm{CDC}$ and WHO, but all three classification systems were associated with increasing body fat and cardiometabolic risk factors from normal weight to obesity. The persistence of childhood obesity in adulthood [29, 30] highlights the need for an early identification of excess weight onset. A consensus on childhood obesity assessment is essential.

\section{Abbreviations}

BMI: Body mass index; CBHSSJB: Cree Board of Health and Social Services of James Bay; CDC: Centers for Disease Control and Prevention; DBP: Diastolic blood pressure; HDL-C: High-density lipoprotein cholesterol; HOMA2IR: Homeostatic model assessment 2 of insulin resistance; IOTF: International Obesity Task Force; SBP: Systolic blood pressure; TG: Triglycerides; WC: Waist circumference; WHO: World Health Organization; Kw: Weighted kappa

\section{Acknowledgments}

The authors are grateful to the Eeyou communities for their participation and all staff from the communities, the Cree Board of Health and Social Services of James Bay (CBHSSJB) and universities who made this project possible.

\section{Funding}

This scientific communication is a report from the Nituuchischaayihtitaau Aschii: A Multi-Community Environment-and-Health Study in Eeyou Istchee was supported by the Eeyou people from northern Quebec, the Cree First Nations, and the Cree Board of Health and Social Services of James Bay (CBHSSJB) through financial support from Niskamoon Corporation (agreement number 2005.06).

\section{Availability of data and materials}

Data are not publicly available. More information is available from the corresponding author on request.

\section{Authors' contributions}

ASJ, SM, PA and ML conceptualized the current analysis, ASJ and SM analyzed the data, and ASJ wrote the first manuscript draft. All authors contributed to data interpretation and critical article revision for important intellectual content and approved the final manuscript version.

\section{Competing interest}

The authors declare that they have no competing interests.

\section{Ethics approval and consent to participate}

Participation was voluntary, and written informed consent was given by one of the child's parents or guardian. Ethics approval was obtained from Cree Board of Health and Social Services of James Bay and Laval, McGill and McMaster Universities.
Consent for publication

Not applicable

\section{Publisher's Note}

Springer Nature remains neutral with regard to jurisdictional claims in published maps and institutional affiliations.

\section{Author details}

${ }^{1}$ Population Health and Optimal Health Practices Research Unit, CHU de Québec - Université Laval, Hôpital du Saint-Sacrement, 1050 Chemin Sainte-Foy, Québec, QC G1S 4L8, Canada. ${ }^{2}$ Institut National de Santé Publique du Québec (INSPQ), 945 Avenue Wolfe, Québec, QC G1V 5B3, Canada. ${ }^{3}$ Department of Social and Preventive Medicine, Faculty of Medecine, Université Laval, 1050 Avenue de la Médecine, Local 2428, Québec, QC G1V 06A, Canada.

Received: 30 November 2016 Accepted: 15 November 2017

Published online: 22 November 2017

References

1. World Health Organization. Obesity: preventing and managing the global epidemic report of a WHO consultation. Geneva: World Health Organization; 2000 .

2. Cali AM, Caprio S. Obesity in children and adolescents. J Clin Endocrinol Metab. 2008;93(11 Suppl 1):S31-S316.

3. Reilly JJ, Kelly J. Long-term impact of overweight and obesity in childhood and adolescence on morbidity and premature mortality in adulthood: systematic review. Int J Obes. 2011;35(7):891-8.

4. Wang Y, Lobstein T. Worldwide trends in childhood overweight and obesity. Int J Pediatr Obes. 2006;1(1):11-25.

5. Katzmarzyk PT. Obesity and physical activity among aboriginal Canadians. Obesity (Silver Spring). 2008;16(1):184-90.

6. Shields M. Overweight and obesity among children and youth. Health Rep. 2006;17(3):27-42.

7. Cole TJ, Bellizzi MC, Flegal KM, Dietz WH. Establishing a standard definition for child overweight and obesity worldwide: international survey. BMJ. 2000; 320(7244):1240-3.

8. Kuczmarski RJ, Ogden CL, Guo SS, et al. CDC growth charts for the United States: methods and development. Vital Health Stat 11. 2000;2002(246):1-190

9. de Onis M, Onyango AW, Borghi E, et al. Development of a WHO growth reference for school-aged children and adolescents. Bull World Health Organ. 2007:85(09):660-7.

10. Edwards J, Evans J, Brown AD. Using routine growth data to determine overweight and obesity prevalence estimates in preschool children in the Capital Health region of Alberta. Can J Public Health. 2008;99(2):91-4.

11. Flegal KM, Ogden $C L$, Wei $R$, et al. Prevalence of overweight in US children: comparison of US growth charts from the Centers for Disease Control and Prevention with other reference values for body mass index. Am J Clin Nutr. 2001;73(6):1086-93.

12. Keke LM, Samouda H, Jacobs J, et al. Body mass index and childhood obesity classification systems: a comparison of the French, international obesity task force (IOTF) and World Health Organization (WHO) references. Rev Epidemiol Sante Publique. 2015;63(3):173-82.

13. Twells LK, Newhook LA. Obesity prevalence estimates in a Canadian regional population of preschool children using variant growth references. BMC Pediatr. 2011:11:21.

14. Medehouenou TC, Ayotte P, St-Jean A, et al. Overweight and obesity prevalence among school-aged Nunavik Inuit children according to three body mass index classification systems. J Adolesc Health. 2015;57(1):31-6.

15. Nieboer E, Dewailly E, Johnson-Down L, et al. Nituuchischaayihtitaau Aschii multi-community environment-and-health study in Eyou Istchee, 20052009: final technical report. Chisabi: Cree Board of Health and Socia Services of James Bay; 2013.

16. A SAS program for the CDC growth charts (ages 0 to $<20$ years). Centers for Disease Control and Prevention website. Available at: http://www.cdc.gov/ nccdphp/dnpao/growthcharts/resources/sas.htm. Accessed March 7, 2016

17. Hemmelgarn BR, McAllister FA, Myers MG, et al. The 2005 Canadian hypertension education program recommendations for the management of hypertension: part I - blood pressure measurement, diagnosis and assessment of risk. Can J Cardiol. 2005;21(8):645-56.

18. Levy JC, Matthews DR, Hermans MP. Correct homeostasis model assessment (HOMA) evaluation uses the computer program. Diabetes Care. 1998;21(12):2191-2. 
19. Landis JR, Koch GG. The measurement of observer agreement for categorical data. Biometrics. 1977;33(1):159-74.

20. Al-Sendi AM, Shetty P, Musaiger AO. Prevalence of overweight and obesity among Bahraini adolescents: a comparison between three different sets of criteria. Eur J Clin Nutr. 2003:57(3):471-4.

21. Gonzalez-Casanova I, Sarmiento OL, Gazmararian JA, et al. Comparing three body mass index classification systems to assess overweight and obesity in children and adolescents. Rev Panam Salud Publica. 2013;33(5):349-55.

22. Kelishadi R, Ardalan G, Gheiratmand R, et al. Thinness, overweight and obesity in a national sample of Iranian children and adolescents: CASPIAN study. Child Care Health Dev. 2008:34(1):44-54.

23. Ma YN, Chen T, Wang D, et al. Prevalence of overweight and obesity among preschool children from six cities of northeast China. Arch Med Res. 2011:42(7):633-40.

24. Minghelli B, Nunes C, Oliveira R. Body mass index and waist circumference to define thinness, overweight and obesity in Portuguese adolescents: comparison between CDC, IOTF, WHO references. Pediatr Endocrinol Rev. 2014;12(1):35-41.

25. Ramirez E, Grijalva-Haro MI, Ponce JA, Valencia ME. Prevalence of overweight and obesity in northwest Mexico by three references of body mass index: differences in classification. Arch Latinoam Nutr. 2006;56(3):251-6.

26. Shields M, Tremblay MS. Canadian childhood obesity estimates based on WHO, IOTF and CDC cut-points. Int J Pediatr Obes. 2010;5(3):265-73.

27. Roberts KC, Shields M, de Groh M, et al. Overweight and obesity in children and adolescents: results from the 2009 to 2011 Canadian health measures survey. Health Rep. 2012;23(3):37-41.

28. Daniels SR. Complications of obesity in children and adolescents. Int J Obes. 2009:33(Suppl 1):S60-5.

29. Serdula MK, Ivery D, Coates RJ, et al. Do obese children become obese adults? A review of the literature. Prev Med. 1993;22(2):167-77.

30. Whitaker RC, Wright JA, Pepe MS, et al. Predicting obesity in young adulthood from childhood and parental obesity. N Engl J Med. 1997; 337(13):869-73.

\section{Submit your next manuscript to BioMed Central and we will help you at every step:}

- We accept pre-submission inquiries

- Our selector tool helps you to find the most relevant journal

- We provide round the clock customer support

- Convenient online submission

- Thorough peer review

- Inclusion in PubMed and all major indexing services

- Maximum visibility for your research

Submit your manuscript at www.biomedcentral.com/submit

) Biomed Central 